\title{
Flower Pollination Algorithm with Bee Pollinator for Cluster Analysis
}

\author{
Rui Wang ${ }^{1}$, Yongquan Zhou ${ }^{1,2}$, Shilei Qiao ${ }^{1}$, Kang Huang ${ }^{1}$ \\ ${ }^{1}$ College of Information Science and Engineering,Guangxi University for Nationalities, Nanning 530006, China \\ ${ }^{2}$ Guangxi High School Key Laboratory of Complex System and Computational Intelligence, \\ Nanning 530006, China \\ E-mail:yongquanzhou@126.com
}

\begin{abstract}
Clustering is a popular data analysis and data mining technique. The K-Means clustering algorithm is one of the most commonly used method. However, it highly depends on the initial solution and is easy to trap into the local optimal. For overcoming these disadvantages of the k-means method, Flower Pollination Algorithm with Bee Pollinator is proposed. Discard pollen operator and crossover operator are applied to increase diversity of the population, and local searching ability is enhanced by using elite based mutation operator. Ten data sets are selected to evaluate the performance of proposed algorithm. Compared with DE, CS, ABC, PSO, FPA and K-Means, the experiment results show that Flower Pollination Algorithm with Bee Pollinator has not only higher accuracy but also higher level of stability. And the faster convergence speed can also be validated by statistical results.
\end{abstract}

Keywords: flower pollination algorithm; discard pollen operator; elite based mutation operator; crossover operator; clustering problem.

\section{Introduction}

Data clustering is the process of grouping together similar multi-dimensional data vectors into a number of clusters. Clustering algorithms have been applied to a wide range of problems, including exploratory data analysis, data mining [1], image segmentation [2] and mathematical programming [3]. Clustering techniques have been used successfully to address the scalability problem of machine learning and data mining algorithms, where prior to, and during training, training data is clustered, and samples from these clusters are selected for training, thereby reducing the computational complexity of training process, and even improving generalization performance [4] [5].

Clustering algorithms can be grouped into two main classes of algorithms, namely hierarchical and partitional. The k-means clustering method [6] is one of the most commonly used partitional methods. However the results of k-means solving the clustering problems highly depend on the initial solution and it is easy to fall into local optimal solutions. For overcoming this problem, many scholars began to solve the clustering problem using meta-heuristic algorithms. Nikam et al have proposed an efficient hybrid evolutionary algorithm based on combining ACO and SA (simulated annealing algorithm, 1989 [7] for clustering problem [8] [9]. In 1991, Colorni A et al have presented ant colony optimization (ACO) algorithm based on the behavior of ants seeking a path between their colony and a source of food. Then P. S. Shelokar and Y. Kao solved the clustering problem using the ACO algorithm [10] [11]. Kennedy $\mathbf{J}$ and Eberhart RC have proposed particle swarm optimization (PSO) algorithm which simulates the movement of organisms in bird flock or fish school in 1995 [12]. The algorithm also has been adopted to solve this problem by M. Omran and V. D. Merwe [13] [14]. Kao et al have presented 
a hybrid approach according to combination of the k-means algorithm, Nelder-Mead simplex search and PSO for clustering analysis [15]. Kevin et al have used an evolutionary-based rough clustering algorithm for the clustering problem [16].

In this paper, a variant of the flower pollination algorithm is used to solve the data clustering problem. Flower Pollination Algorithm (proposed by Yang in 2012) [17] is a new population-based intelligent optimization algorithm by simulating flower pollination behavior in nature. And it has been extensively researched in last two years by scholars. Yang and Xingshi He have used FPA to solve multi-objective optimization problem in 2013[18]. Marwa Sharawi has applied FPA for solving Wireless Sensor Network Lifetime global optimization in 2014 [19]. Osama Abdel-Raouf has used an improved FPA to solve Sudoku Puzzles in 2014 [20]. And FPA has been used to solve Large Integer Programming Problems by Ibrahim El-henawy in 2014 [21].

The remainder of this paper is organized as follows. Section 2 introduces the mathematical model of clustering; Section 3 describes the principle of basic flower pollination algorithm; while section 4 specified implementation procedure of our proposed Flower Pollination Algorithm with Bee Pollinator (BPFPA); in section 5, Series of comparison experiments are conducted; Result analysis will be given in Section 6; Our conclusion and future works are described in section 7; and acknowledgements are shown in section 8 .

\section{The mathematical model of clustering}

\subsection{Mathematical definition of data clustering}

The goal of data clustering is grouping data into a number of clusters, clustering problems can be seen in practice frequently. In this subsection, a mathematical definition of data clustering is presented. In order to explain the definition clearly, we supposed that there exists a data set $D=\left\{d_{1}, d_{2}, \ldots d_{n}\right\}$. And each individual $d_{i}(i=1,2, \ldots, n)$ has many features. If the dimension is $m$, each individual can be shown as $d_{i}=\left(l_{1}, l_{2}, \ldots, l_{m}\right)$. Data clustering is a process which can classify the given data set $D$ into a certain numbers of clusters $G_{1}, G_{2}, \ldots, G_{K}$ (assume $K$ clusters) based on the similarity of individuals. And $G_{1}, G_{2}, \ldots, G_{K}$ should satisfy the following formulas:

1). $G_{i} \neq \varnothing, i=1,2, \ldots, K$.

2). $G_{i} \cap G_{j}=\varnothing, i, j=1,2, \ldots, K, i \neq j$.

3). $\bigcup_{i=1}^{K} G_{i}=\left\{d_{1}, d_{2}, \ldots, d_{n}\right\}$.

\subsection{The principle of data clustering}

In the clustering process, if the given data set $D$ should be divided into $K$ clusters $\left(G_{1}, G_{2}, \ldots, G_{K}\right)$, and each cluster must have one center $c_{j}(j=1,2, \ldots, K)$. It is supposed that $C=\left(c_{1}, c_{2}, \ldots, \mathrm{c}_{K}\right)$ are the centers of $\left(G_{1}, G_{2}, \ldots, G_{K}\right)$. Where $c_{j}$ is the center of subset $G_{j}$.

The main idea of clustering is to define $K$ centers, one for each cluster. These centers should be placed in a crafty way, because different location will causes different result. Therefore, the better choice is to place them as far away from each other as possible. In this paper, we will use Euclidian metric as a distance metric. The expression is given as follows: 


$$
d\left(d_{i}, c_{j}\right)=\sqrt{\sum_{k=1}^{m}\left(d_{i k}-c_{j k}\right)^{2}}
$$

where $d_{i}(i=1,2, \ldots, n)$ is an individual in the given data set $D, m$ is the number of individual features; $c_{j}(j=1,2, \ldots, K)$ is the center of $j$ th subset. Because individual has $m$ features, $c_{j}$ can be presented by $\left(c_{j, 1}, c_{j, 2}, \ldots c_{j, m}\right)$. In order to confirm which subset $d_{i}$ belongs to, the distances between $d_{i}$ and $c_{j}(j=1,2, \ldots, K)$ should be calculated via (1). If the distance between $d_{i}$ and $c_{\text {best }}($ best $=1,2, \ldots, K)$ is smaller than the distances between $d_{i}$ and other centers (except $c_{\text {best }}$ ), we can make the decision that $d_{i}$ should belongs to $G_{b e s t}$. For example, if the value of $d\left(d_{1}, c_{2}\right)$ is smaller than $d\left(d_{1}, c_{j}\right),(j \neq 2)$, we can draw the conclusion that $d_{1}$ should be distributed to $G_{1}$.

\subsection{The performance evaluation function of data clustering}

In this paper, data clustering problem is solved by population based algorithm (Flower Pollination Algorithm and Flower Pollination Algorithm with Bee Pollinator). For explaining the evaluation process explicitly, we suppose that given data set $D$ should be divided into $K$ subsets. And the dimension of individual of data set $D$ is $m$. In order to optimize the coordinates of centers of $K$ subsets, it is easily to find that the dimension of solution should be $K * m$. The individual in the population can be described as $s=\left(c_{1}, c_{2}, \ldots, \mathrm{c}_{K}\right)$. A great classification should minimize the sum of distances value. So we should try to minimize the distance between individual $d_{i}$ and the center $\left(c_{j}\right)$ of subset it belongs to. Finally, the proposed algorithm aims at minimizing the objective function, which can be expressed as following:

$$
f(D, C)=\sum_{i=1}^{n} \operatorname{Min}\left\{\left\|d_{i}-c_{k}\right\| \mid k=1,2, \cdots, K\right\}
$$

where $D=\left(d_{1}, d_{2}, \ldots, d_{n}\right)$ is the given data set, $C=\left(c_{1}, c_{2}, \ldots, c_{K}\right)$ is the centers of subsets $\left(G_{1}, G_{2}, \ldots, G_{K}\right)$.

\section{Flower Pollination Algorithm ( FPA )}

Flower Pollination Algorithm (FPA) was founded by Yang in the year 2012. Inspired by the flow pollination process of flowering plants are the following rules [22] [17]:

Rule 1: Biotic and cross-pollination can be considered as a process of global pollination process, and pollen-carrying pollinators move in a way that obeys Lévy flights.

Rule 2: For local pollination, a biotic and self-pollination are used.

Rule 3: Pollinators such as insects can develop flower constancy, which is equivalent to a reproduction probability that is proportional to the similarity of two flowers involved.

Rule 4: The interaction or switching of local pollination and global pollination can be controlled by a switch probability, with a slight bias toward local pollination.

In order to formulate updating formulas, we have to convert the aforementioned rules into updating equations. For example, in the global pollination step, flower pollen gametes are carried by pollinators such as insects, and pollen can travel over a long distance because insects can often fly and move in a much longer range. Therefore, Rule 1 and flower constancy can be represented mathematically as:

$$
x_{i}^{t+1}=x_{i}^{t}+\gamma L(\lambda)\left(x_{i}^{t}-B\right)
$$


Where $x_{i}^{t}$ is pollen $i$ or solution vector $x_{i}$ at iteration $t$, and $B$ is the current best solution found among all solutions at the current generation/iteration. Here $\gamma$ is a scaling factor to control the step size.

In addition, $L(\lambda)$ is the parameter that corresponds to the strength of the pollination, which essentially is also the step size. Since insects may move over a long distance with various distance steps, we can use a Lévy flight to imitate this characteristic efficiently. That is, we draw $L>0$ from a Levy distribution:

$$
L \sim \frac{\lambda \Gamma(\lambda) \sin (\pi \lambda / 2)}{\pi} \frac{1}{S^{1+\lambda}},\left(S>>S_{0}>0\right)
$$

Here, $\Gamma(\lambda)$ is the standard gamma function, and this distribution is valid for large steps $s>0$. Then, to model the local pollination, both Rule 2 and Rule 3 can be represented as

$$
x_{i}^{t+1}=x_{i}^{t}+U\left(x_{j}^{t}-x_{k}^{t}\right)
$$

Where $x_{j}^{t}$ and $x_{k}^{t}$ are pollen from different flowers of the same plant species. This essentially imitates the flower constancy in a limited neighborhood. Mathematically, if $x_{j}^{t}$ and $x_{k}^{t}$ comes from the same species or selected from the same population, this equivalently becomes a local random walk if we draw $U$ from a uniform distribution in [0,1]. Though flower pollination activities can occur at all scales, both local and global, adjacent flower patches or flowers in the not-so-far-away neighborhood are more likely to be pollinated by local flower pollen than those faraway. In order to imitate this, we can effectively use the switch probability like in Rule 4 or the proximity probability $p$ to switch between common global pollination to intensive local pollination. To begin with, we can use a naïve value of $p=0.5$ as an initially value. A preliminary parametric showed that $p=0.8$ might work better for most applications [17].

\section{Flower Pollination Algorithm with Bee Pollinator ( BPFPA )}

For strengthening the global searching and local searching abilities, three optimization strategies are applied to the basic flower pollination algorithm (FPA). As we all know that honey bees usually act as pollinators of flower plants in nature. And there exists a discard solution operator in artificial bee colony algorithm $(\mathrm{ABC})$ [23]. In this paper, that operator is selected to enhance the global searching ability of proposed Flower Pollination Algorithm with Bee Pollinator. Other two optimization strategies (Elite Based Mutation Operator and Crossover Operator) used in local search process to enhance its local searching ability.

\subsection{Discard Pollen Operator}

In natural, Bees usually act as the pollinators of flowers. And there exists a discard solution operator in $\mathrm{ABC}$ [23] to enhance the diversity of population and the global searching ability. In ABC, limit is an important controls parameter. If a solution can't be improved after limit generation continuously, and it is not the global best in the population, we should discard it and generate a new solution in a random way.

Honey bees use levy flight to do global search in proposed Flower Pollination Algorithm with Bee Pollinator. And discard pollen operator is chosen to enhance its global searching ability. If a pollen is not improved after limit generations continuously, and it is not the global best, we should discard it and generate a new pollen use simplex method. The steps of simplex method [26] [25] are shown as follows (Fig.1): 


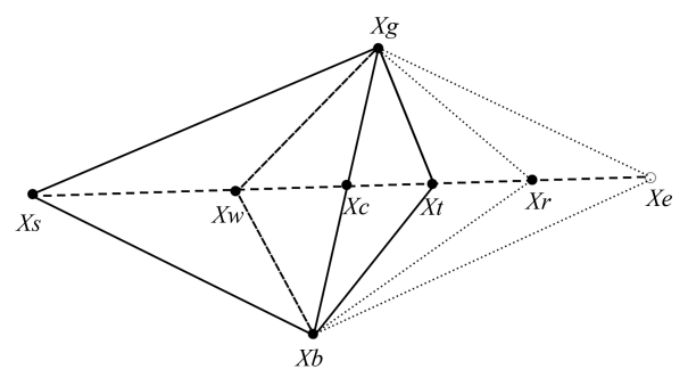

Fig.1 The simplex method searches the difference points

Step 1: Evaluate all the solutions in the population, and choose the global best $x_{g}$, and the second best $x_{b}$. It is supposed that $x_{s}$ is the pollen should be discarded. $f\left(x_{g}\right), f\left(x_{b}\right), f\left(x_{s}\right)$ are the fitness value of $x_{g}, x_{b}, x_{s}$.

Step 2: Find the center point $x_{c}$ between $x_{g}$ and $x_{s}$ by the expression below:

$$
x_{c}=\frac{x_{g}+x_{b}}{2}
$$

Step 3: Carry out the reflecting operation by expression below:

$$
x_{r}=x_{c}+\alpha\left(x_{c}-x_{s}\right)
$$

where $x_{r}$ is the reflecting point of $x_{s}$. $\alpha$ is the reflecting coefficient, which is set to $1 \mathrm{in}$ this paper.

Step 4: If the fitness value of $x_{r}$ is smaller than that of $x_{g}\left(f\left(x_{r}\right)<f\left(x_{g}\right)\right)$, extension operation should be carried out according to the expression below:

$$
x_{e}=x_{c}+\gamma\left(x_{r}-x_{c}\right)
$$

where $x_{e}$ is the extension point. And the extension coefficient $\gamma$ should be set to 2 . If the fitness value of $x_{e}$ is smaller than the fitness of $x_{g}, x_{s}$ should be replaced by $x_{r}$, otherwise $x_{r}$ will be used to substitute $x_{s}$.

Step 5: If $f\left(x_{r}\right)>f\left(x_{s}\right)$, the compression operation should be used via expression below:

$$
x_{t}=x_{c}+\beta\left(x_{s}-x_{c}\right)
$$

where $x_{t}$ is the compression point of $x_{s}$, the compression coefficient $\beta$ should be set to 0.5 . If $f\left(x_{t}\right)<f\left(x_{s}\right), x_{s}$ should be replaced by $x_{t}$.

Step 6: If $f\left(x_{s}\right)>f\left(x_{r}\right)>f\left(x_{g}\right)$, shrink operation should be carried out according to expression below:

$$
x_{w}=x_{c}-\beta\left(x_{s}-x_{c}\right)
$$

where $x_{w}$ is the shrink point. Shrink coefficient $\beta$ is set to 0.5 in this paper. If $f\left(x_{w}\right)<f\left(x_{s}\right)$, $x_{s}$ should be replaced by $x_{w}$, otherwise, we use $x_{r}$ to substitute $x_{s}$.

In global search process of Flower Pollination Algorithm with Bee Pollinator, the discard pollen operator is used to jump out local minima, which enhance its global searching ability significantly. And simplex method is used to generate new pollen to substitute the bad one. New pollen generated by simplex method usually has a better fitness, which can speed up the convergence speed.

\subsection{Elite Based Mutation Operator}

Flower Pollination Algorithm (developed by Yang) carries out local search in a similar way to the step in differential evolution (DE). And experiment results show that local search ability of DE is limited. Thus, we add Elite Based Mutation Operator to local search process to enhance its exploitation ability. And that operator can be expressed in the following formula: 


$$
X_{i, G+1}=X_{i, G}+\alpha\left(X_{b e s t}-X_{i, G}\right)+\beta\left(X_{p, G}-X_{q, G}\right)
$$

where $X_{b e s t}$ is the best vector of the whole population, $p, q \in[1, n], n$ is the population size. $\alpha, \beta$ obey uniform distribution. The exploitation ability of proposed algorithms is enhanced by this mutation operator.

\subsection{Crossover Operator}

In subsection 4.2, Elite Based Mutation Operator is used to the local search process. EBMO can increase its exploitation ability, and it can also speed up the convergence speed. But another aspect should be taken into account. EBMO make it easier to trap in local minima. So crossover operator is chosen to increase the diversity of population. And the crossover operator can be expressed in the following formula:

$$
X_{i, j}=\left\{\begin{array}{l}
X_{i, j}, \operatorname{rand}_{i, j}[0,1]<\mathrm{C}_{r} \\
X_{k, j}, k \neq i \text { else }
\end{array} \quad i, k=1,2,3 \ldots n ; j=1,2, \ldots d .\right.
$$

where $X_{k, j}$ is the $j$ th variable of $k$ th pollen; $C_{r}$ is the crossover rate, which is set to 0.15 in this paper. The crossover operator helps to increase the diversity of population in local search process, and avoid trapping into local optimal. Specific implementation steps of Flower Pollination Algorithm with Bee Pollinator (BPFPA) for clustering can be summarized in the pseudo code shown in Algorithm 1.

Algorithm1 .Flower Pollination Algorithm with Bee Pollinator for clustering problem

Initialize a population $(X)$ of $n$ flowers/pollen gametes with random solutions.

Each flower represents all center points of clusters.

Initialize the vector counter $=z \operatorname{eros}(1, n)$; it is used to record the generation of unimproved flowers.

Set limit $=10$;

Find the best solution $\boldsymbol{B}$ in the initial population

Define a switch probability $p \in(0,1)$

Define a stopping criterion (either a fixed number of generations/iterations or accuracy)

while $(t<$ MaxGeneration $)$

for $\mathrm{i}=1: n($ all $n$ flowers in the population)

if rand $<p$

(Global search process)

Draw a (d-dimensional) step vector $L$ which obeys a Lévy distribution.

Global pollination via equation(3) and get new solution $x_{i}$.

if counter $(1, i)=$ limit

Generate a new solution via simplex methods (discussed in section 4.1);

counter $(1, i)=0$;

end if

else

(Local search process)

Carry out Elite Based Mutation Operation via equation(11).

Carry out Crossover Operation via equation(12) .

end if 
Evaluate the new solution $x_{i}$ via equation (2);

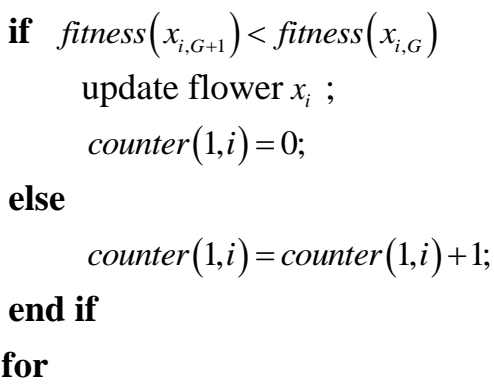

\section{end for}

Find the current best solution $\boldsymbol{B}$

end while

Output the best solution found.

\section{Simulation Experiments}

\subsection{Experimental setup}

All of the algorithm was programmed in MATLAB R2012a, numerical experiment was set up on AMD Athlont (tm) II*4640 processor and 2 GB memory.

\subsection{Numerical Comparison of each algorithm performance}

The proposed Flower Pollination Algorithm with Bee Pollinator compared with mainstream swarm intelligence algorithms DE [24], CS [27], PSO [12], ABC [23], FPA [17] and K-means [6], respectively using the mean and standard deviation to compare their optimal performance. The setting values of algorithm control parameters of the mentioned algorithms are given below.

DE setting: $F=0.5$ and $C R=0.9$ in accordance with the suggestions given in [24], the population size is 50 .

CS setting: $p_{a}=0.25$ have been used as recommended in [27], the population size is 50 .

PSO setting: weight factor $\omega=0.7298, c_{1}=c_{2}=1.4962$ [12], the population size is 50 .

$\mathrm{ABC}$ setting: limit $=5 D$ has been used as recommended in [23], the population size is 50 .

FPA setting: switch probability $p=0.8$ in accordance with the suggestions given in [17], the population size is 50.And the maximum generations of all algorithms are set 200.

In this section, ten data sets are selected to testify the effectiveness of proposed algorithm, including two artificial data sets and eight real-life data sets (selected from the UCI machine learning repository [28]). There are artificial set one, artificial set two, Iris, Wisconsin breast cancer, Contraceptive Method Choice (CMC), Statlog (Heart), Balance Scale, Seeds, Haberman's Survival, Wine.

For these data sets, each algorithm is applied 20 times individually with random initial solution. For art1 and art2 data sets, once the randomly generated parameters are determined, the same parameters are used to test the performance of three algorithms. The best value, the worst value, the mean value and standard deviation are recorded in Table 1 to 10. Bold and italicized results mean that BPFPA is better, while underlined results mean that other algorithm is better. The results are kept four digits after the decimal point.

5.2.1 Artificial data set one

Artificial data set one $(N=250, d=3, K=5)$. This is a three-featured problem with five classes, 
where every feature of the classes was distributed according to Class 1-Uniform $(85,100)$, Class 2-Uniform (70,85), Class 3-Uniform (55,70), Class 4-Uniform (40,55), Class 5-Uniform $(25,40)$ [29].The comparison of algorithms for artificial data set one is listed in Table 1. And the convergence curves of algorithms are shown in Fig.2. In Fig.3, it is the result of anova test for artificial data set one.

Table 1. Results obtained by the algorithms for 20 different runs on Artificial data set one

\begin{tabular}{ccccc}
\hline Algorithm & Best & Worst & Mean & Std. \\
\hline BPFPA & $\mathbf{1 7 1 8 . 2 5 3 8}$ & 2386.5104 & $\mathbf{1 7 8 3 . 5 1 4 7}$ & 200.9330 \\
FPA & 1977.7401 & 2418.7069 & 2173.4082 & 115.4865 \\
CS & 1725.2482 & $\underline{1995.5823}$ & 1806.2505 & $\underline{75.4400}$ \\
PSO & 1718.2538 & 2444.8930 & 2153.7595 & 344.4796 \\
ABC & 1788.2069 & 2059.1212 & 1899.4503 & 77.49667 \\
DE & 1718.4592 & 2495.0929 & 1974.5335 & 346.0330 \\
K-means & 1747.3859 & 2507.9091 & 1991.9351 & 342.2974 \\
\hline
\end{tabular}

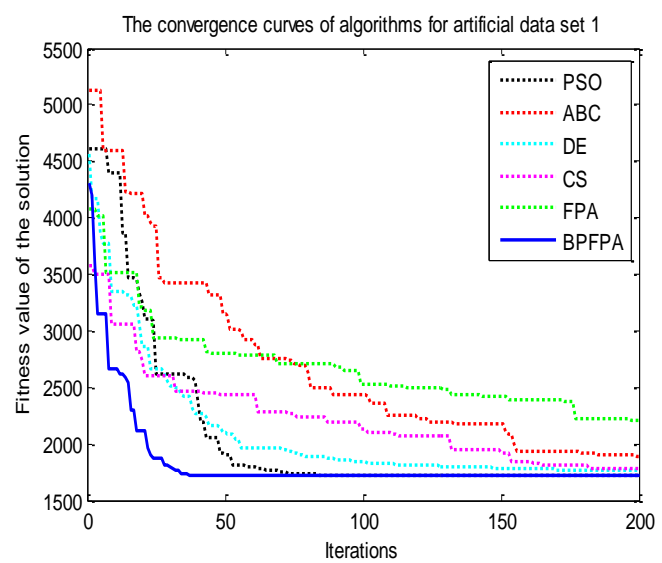

Fig.2 The convergence curves of algorithms for art1

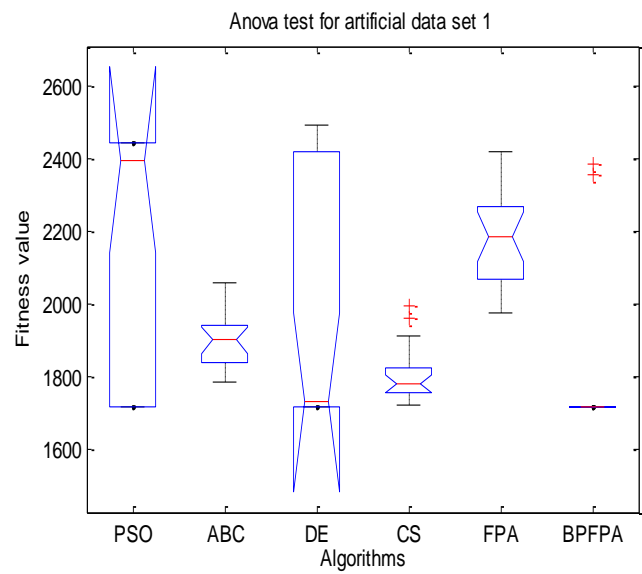

Fig.3 The anova test of algorithms for art1

From Table 1, the best value and the mean value of BPFPA are much better than those of other algorithms mentioned in this paper can be found. Though the standard deviation of CS is smaller, the mean value of CS is not good enough. Six convergence curves can be seen in Fig.2, which is the comparison for artificial data set one. We can discover that the curve of BPFPA is much smoother than other algorithms. And its convergence speed is faster. Fig. 3 is the anova test for data set art1.We can find that the stability of BPFPA is well, the reason of its large deviation is two outliers shown in Fig.3.

\subsubsection{Artificial data set two}

Artificial data set two $(N=600, d=2, K=4)$. This is a two-featured problem with four unique classes. A total of 600 patterns were drawn from four independent bivariate normal distributions, where classes were distributed according to

$$
N_{2}\left(\mu=\left(\begin{array}{l}
m_{i} \\
0
\end{array}\right), \sum=\left[\left[\begin{array}{lr}
0.5 & 0.05 \\
0.05 & 0.5
\end{array}\right]\right]\right)
$$

$i=1,2,3,4 \quad m_{1}=-3, m_{2}=0, m_{3}=3, m=6$. 
$\mu$ and $\sum$ being mean vector and covariance matrix, respectively [29]. The comparison of algorithms for artificial data set two is listed in Table 2. And the convergence curves of algorithms are shown in Fig.4. In Fig.5, it is the result of anova test for artificial data set two.

Table 2. Results obtained by the algorithms for 20 different runs on Artificial data set two

\begin{tabular}{ccccc}
\hline Algorithm & Best & Worst & Mean & Std. \\
\hline BPFPA & $\mathbf{5 1 3 . 9 0 3 5}$ & $\mathbf{5 1 3 . 9 0 3 5}$ & $\mathbf{5 1 3 . 9 0 3 5}$ & $\mathbf{1 . 3 3 E - 1 3}$ \\
FPA & 517.1823 & 568.8652 & 538.6455 & 14.9615 \\
CS & 513.9037 & 515.931 & 514.1077 & 0.5302 \\
PSO & 513.9035 & 513.9035 & 513.9035 & $2.14 \mathrm{E}-08$ \\
ABC & 514.6936 & 534.4247 & 518.3271 & 4.430872 \\
DE & 513.9035 & 514.2079 & 513.9463 & 0.0781 \\
K-means & 525.5957 & 907.1413 & 694.4421 & 191.4831 \\
\hline
\end{tabular}

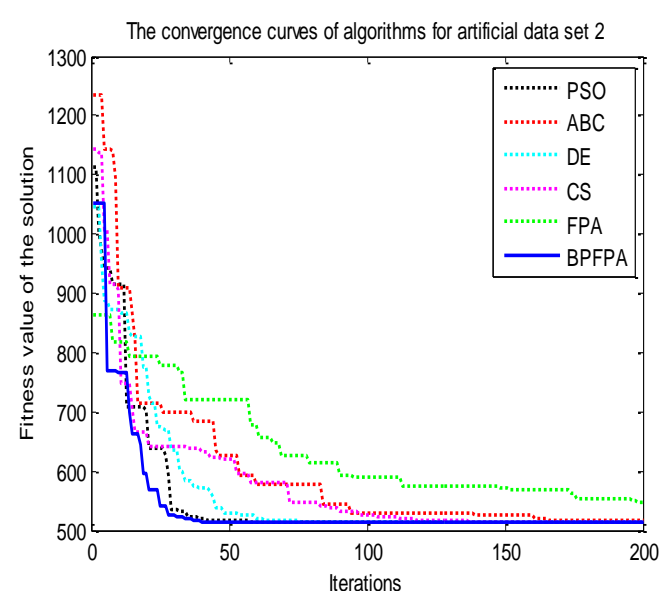

Fig.4 The convergence curves of algorithms for art2

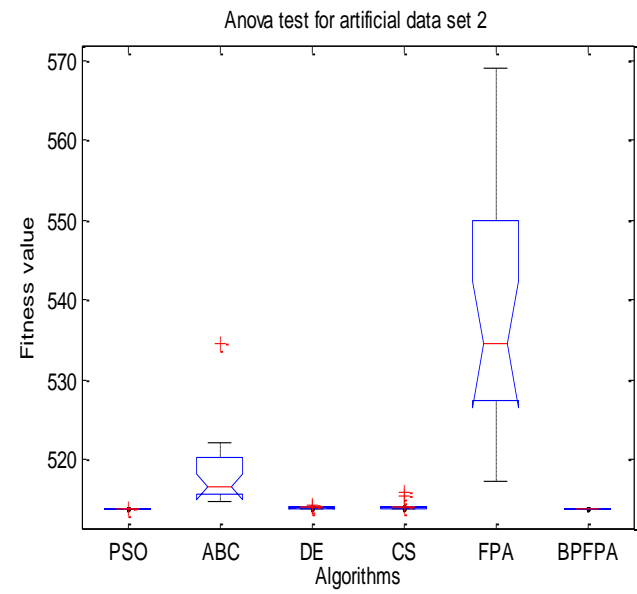

Fig.5 The anova test of algorithms for art2

From Table 2, we can discover that the best value, worst value, mean value and standard deviation of BPFPA are the best in seven algorithms. And six convergence curves for artificial data set two are shown in Fig.4. The curve of BPFPA is smoother than others can be discovered easily. Faster convergence speed of BPFPA can be found as well. Fig.5 shows that BPFPA has a high degree of stability.

\subsubsection{Data set Iris}

Iris data $(N=150, d=4, K=3)$ : this data set with 150 random samples of flowers from the iris species setosa, versicolor, and virginica collected by Anderson (1995). From each species there are 50 observations for sepal length, sepal width, petal length, and petal width in $\mathrm{cm}$. This data set was used by Fisher (1936) in his initiation of the linear-discriminant-function technique [28] [29]. The comparison of algorithms for data set Iris is listed in Table 3. And the convergence curves of algorithms are shown in Fig.6. In Fig.7, it is the result of anova test for Iris. 
Table 3. Results obtained by the algorithms for 20 different runs on Iris

\begin{tabular}{ccccc}
\hline Algorithm & Best & Worst & Mean & Std. \\
\hline BPFPA & $\mathbf{9 6 . 6 5 5 5}$ & $\mathbf{9 6 . 6 5 5 5}$ & $\mathbf{9 6 . 6 5 5 5}$ & $\mathbf{8 . 2 E - 1 3}$ \\
FPA & 97.4786 & 103.5763 & 99.7933 & 1.6846 \\
CS & 96.6569 & 97.1995 & 96.7242 & 0.1260 \\
PSO & 96.6554 & 127.6677 & 105.9627 & 14.5783 \\
ABC & 97.1362 & 100.2921 & 98.1047 & 0.6856 \\
DE & 96.6571 & 105.8550 & 97.5744 & 2.0035 \\
K-means & 97.1901 & 121.3554 & 100.8866 & 8.7805 \\
\hline
\end{tabular}

Table 3 shows that the best value, worst value, mean value and standard deviation of BPFPA are all better than other algorithms. Convergence curves of six algorithms for data set Iris are shown in Fig.6. The curves in Fig.6 show that BPFPA has a faster convergence speed. And the convergence curve of BPFPA is smoother. Higher level of stability of BPFPA can be discovered in Fig.7, which shows the anova test result for Iris.

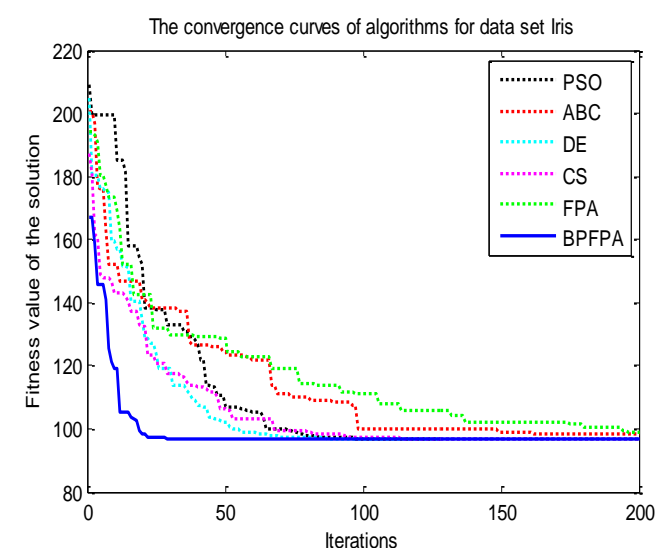

Fig.6 The convergence curves of algorithms for Iris

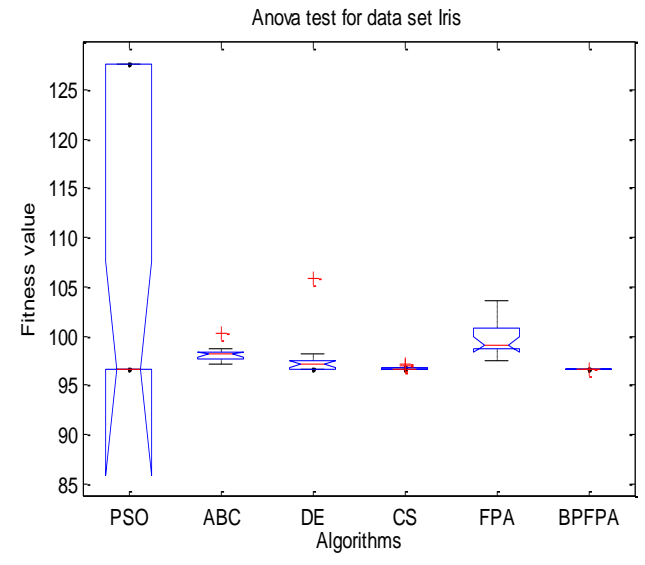

Fig.7 The anova test of algorithms for Iris

\subsubsection{Data set Wisconsin Breast Cancer}

Wisconsin breast cancer $(N=683, d=9, K=2)$ : which consists of 683 objects characterized by nine features: clump thickness, cell size uniformity, cell shape uniformity, marginal adhesion, single epithelial cell size, bare nuclei, bland chromatin, normal nucleoli, and mitoses. There are two categories in the data: malignant (444 objects) and benign (239 objects) [28] [29]. The comparison of algorithms for data set Cancer is listed in Table 4. And the convergence curves of algorithms are shown in Fig.8. In Fig.9, it is the result of anova test for cancer.

Table 4. Results obtained by the algorithms for 20 different runs on Cancer

\begin{tabular}{ccccc}
\hline Algorithm & Best & Worst & Mean & Std. \\
\hline BPFPA & $\mathbf{2 9 6 4 . 3 8 6 9}$ & $\mathbf{2 9 6 4 . 3 8 6 9}$ & $\mathbf{2 9 6 4 . 3 8 6 9}$ & $\mathbf{9 . 0 0 4 1 E - 1 2}$ \\
FPA & 2970.7424 & 3033.8020 & 2987.5210 & 14.3143 \\
CS & 2964.5318 & 2966.6399 & 2964.9456 & 0.4996 \\
PSO & 2964.3871 & 4728.7901 & 3140.8281 & 543.0715 \\
ABC & 2982.8207 & 3190.5028 & 3046.3477 & 48.8843 \\
DE & 2970.7369 & 3119.2544 & 3025.7756 & 49.3631 \\
K-means & 2976.9441 & 2988.4278 & 2983.3164 & 4.8661 \\
\hline
\end{tabular}




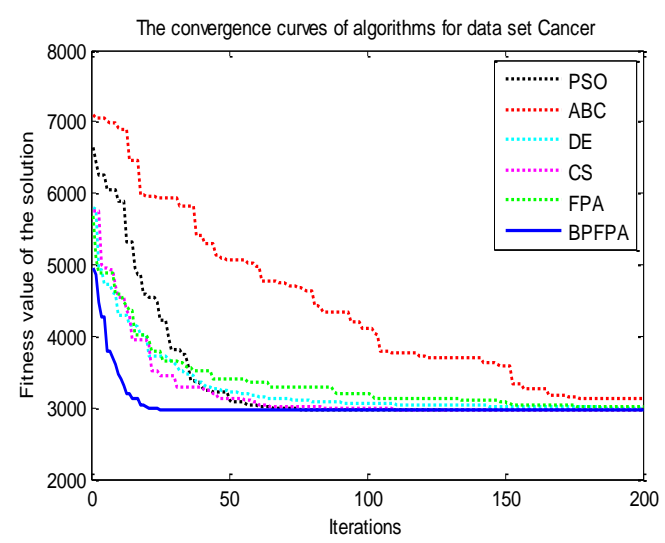

Fig.8 The convergence curves of algorithms for Cancer

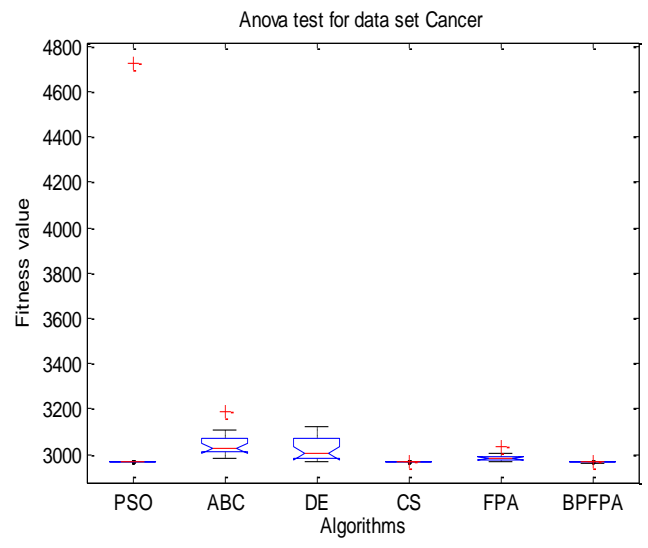

Fig.9 The anova test of algorithms for Cancer

The best value, worst value, mean value and the standard deviation of BPFPA are the best of seven algorithms listed can be easily discovered from Table 4. From the numerical comparison in Table 4, we also can find that CS performs second best. Convergence curves for data set Cancer shown in Fig.8 clearly show that BPFPA has a faster convergence speed. And its high degree of stability can be discovered in Fig.9, which is the anova test for Cancer.

\subsubsection{Data set Contraceptive Method Choice (CMC)}

Contraceptive Method Choice $(N=1473, d=10, K=3)$ : this data set is a subset of the 1987 National Indonesia Contraceptive Prevalence Survey. The samples are married women who were either not pregnant or do not know if they were at the time of interview. The problem is to predict the current contraceptive method choice (no use, long-term methods, or short-term methods) of a woman based on her demographic and socioeconomic characteristics [28] [29]. The comparison of algorithms for data set CMC is listed in Table 5. And the convergence curves of algorithms are shown in Fig.10. In Fig.11, it is the result of anova test for CMC.

Table 5. Results obtained by the algorithms for 20 different runs on CMC

\begin{tabular}{ccccc}
\hline Algorithm & Best & Worst & Mean & Std. \\
\hline BPFPA & $\mathbf{5 6 9 3 . 7 2 3 9}$ & $\mathbf{5 6 9 3 . 7 2 8 3}$ & $\mathbf{5 6 9 3 . 7 2 4 6}$ & $\mathbf{0 . 0 0 1 6}$ \\
FPA & 5796.7620 & 6100.1095 & 5927.0751 & 80.9424 \\
CS & 5704.1720 & 5738.4765 & 5721.1617 & 9.9204 \\
PSO & 5693.7779 & 5694.6504 & 5694.1109 & 0.2400 \\
ABC & 5848.2889 & 6320.9353 & 6041.8898 & 123.7676 \\
DE & 5720.3057 & 6089.6187 & 5811.6135 & 95.0982 \\
K-means & 5703.3444 & 5705.2747 & 5704.0770 & 0.9012 \\
\hline
\end{tabular}




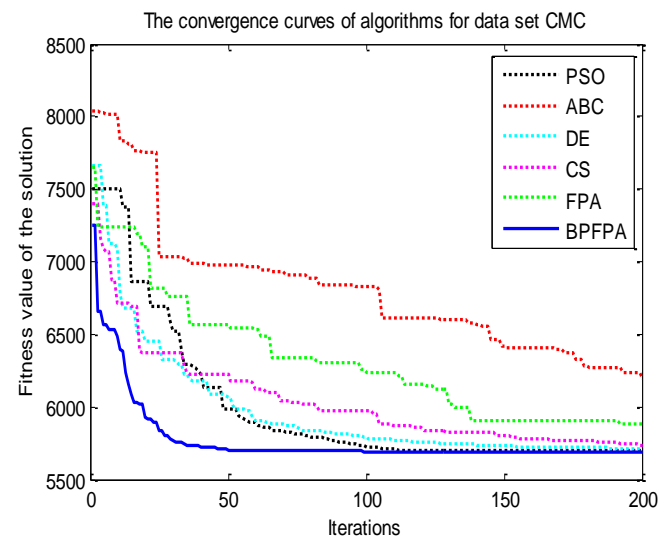

Fig.10 The convergence curves of algorithms for CMC

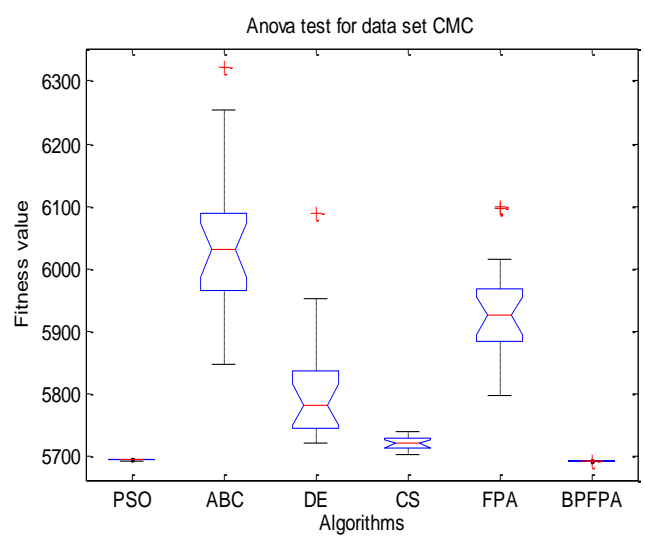

Fig.11 The anova test of algorithms for CMC

From Table 5, we can discover that the best value, worst value, mean value and standard deviation of BPFPA are the best in seven algorithms. ABC performs worst can also be seen from that table. And six convergence curves for data set CMC are shown in Fig.10. The curve of BPFPA is smoother than those of other algorithms can be found easily. Faster convergence speed of BPFPA can be found as well. Fig.11 shows that the stability of BPFPA and PSO are in high level.

\subsubsection{Data set Statlog (Heart)}

Statlog (Heart) data $\operatorname{set}(N=270, d=13, K=2)$ : this data set is a heart disease database similar to a database already present in the repository (Heart Disease databases) but in a slightly different form [28]. The comparison of algorithms for data set Heart is listed in Table 6. And the convergence curves of algorithms are shown in Fig.12. In Fig.13, it is the result of anova test for Heart.

Table 6. Results obtained by the algorithms for 20 different runs on Heart

\begin{tabular}{ccccc}
\hline Algorithm & Best & Worst & Mean & Std. \\
\hline BPFPA & $\mathbf{1 0 6 2 2 . 9 8 2 4}$ & $\mathbf{1 0 6 2 3 . 1 8 2 4}$ & $\mathbf{1 0 6 2 2 . 9 9 2 4}$ & $\mathbf{0 . 0 4 4 7}$ \\
FPA & 10630.4063 & 10646.4106 & 10639.9882 & 4.0078 \\
CS & 10623.3314 & 10624.6019 & 10623.7067 & 0.3131 \\
PSO & 10622.9886 & 10623.4230 & 10623.0434 & 0.1120 \\
ABC & 10673.4980 & 11148.8039 & 10795.8489 & 110.1501 \\
DE & 10628.3745 & 11283.5839 & 10739.1109 & 173.6192 \\
K-means & 10682.0809 & 10700.8385 & 10691.7056 & 8.2080 \\
\hline
\end{tabular}

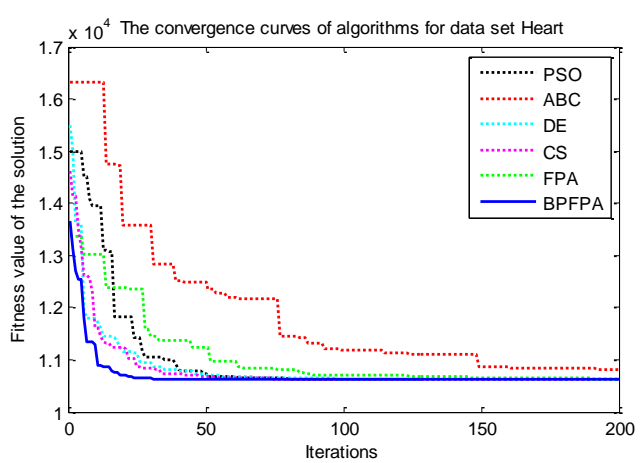

Fig.12 The convergence curves of algorithms for Heart

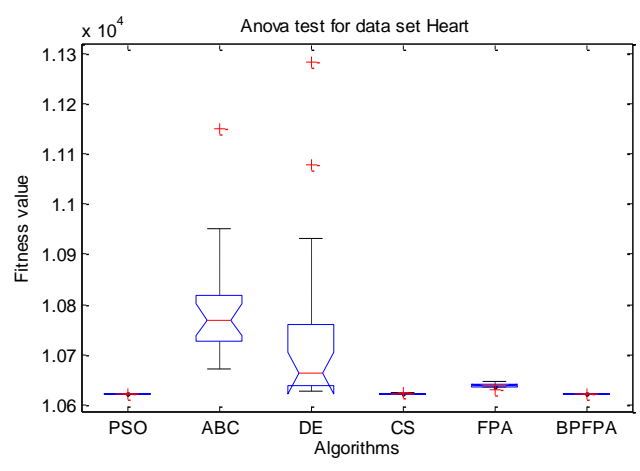

Fig.13 The anova test of algorithms for Heart 
Table 3 shows that all four indicators listed of BPFPA are smaller than those of other algorithms for data set Heart. And PSO performs the second best. ABC shows the weakness of its ability to solving clustering problem for data set Heart. Convergence curves for Heart are shown in Fig.12. The curves in Fig.12 shows the fact that BPFPA has a faster convergence speed. High level of stability of BPFPA can be discovered in Fig.13, which shows the anova test result for Heart.

\subsubsection{Data set Balance Scale}

Balance Scale data $(N=625, d=4, K=3)$ : this data set was generated to model psychological experimental results. Each example is classified as having the balance scale tip to the right, tip to the left, or be balanced. The attributes are the left weight, the left distance, the right weight, and the right distance. The correct way to find the class is the greater of (left-distance * left-weight) and (right-distance* right-weight). If they are equal, it is balanced [28]. The comparison of algorithms for data set Scale is listed in Table 7. And the convergence curves of algorithms are shown in Fig.14. In Fig.15, it is the result of anova test for Scale.

Table 7. Results obtained by the algorithms for 20 different runs on Scale

\begin{tabular}{ccccc}
\hline Algorithm & Best & Worst & Mean & Std. \\
\hline BPFPA & $\mathbf{1 4 2 3 . 8 2 0 4}$ & 1425.7726 & $\mathbf{1 4 2 4 . 3 9 4 9}$ & 0.8473 \\
FPA & 1427.2209 & 1431.0778 & 1428.8768 & 1.0186 \\
CS & 1424.7429 & 1427.7458 & 1426.6609 & 0.9433 \\
PSO & 1423.8204 & $\underline{1425.7714}$ & 1424.8512 & 0.9363 \\
ABC & 1424.6303 & 1427.7899 & 1426.9782 & 0.7207 \\
DE & 1424.1447 & 1432.2318 & 1427.1192 & 2.0181 \\
K-means & 1423.8514 & 1434.0441 & 1426.7539 & 3.1208 \\
\hline
\end{tabular}

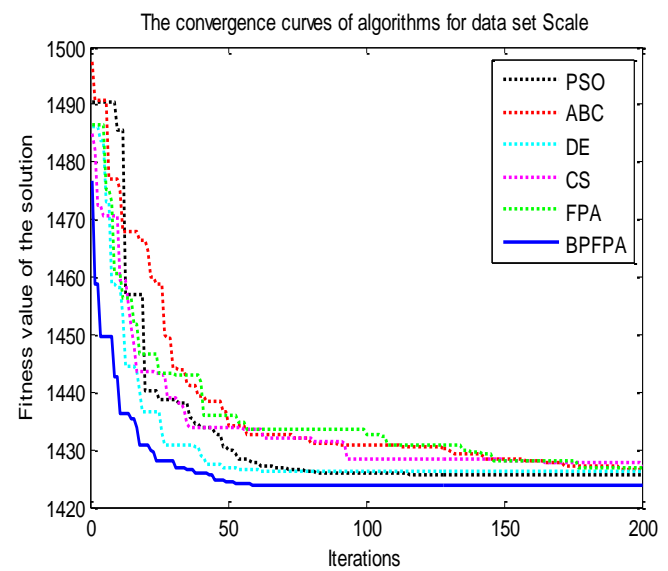

Fig.14 The convergence curves of algorithms for Scale

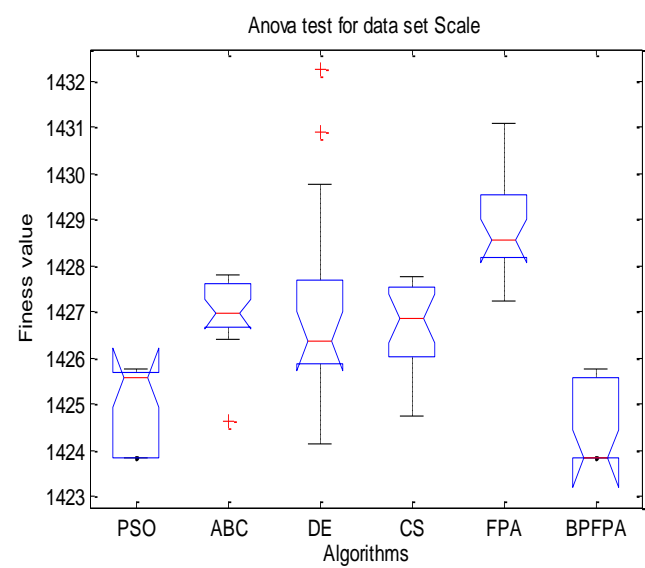

Fig.15 The anova test of algorithms for Scale

From Table 7, the best value and the mean value of BPFPA are the best can be discovered. And the worst value of PSO and the standard deviation of ABC are better than other algorithms. Fig.14 shows the convergence curves of mentioned population based algorithms. Faster convergence speed can be discovered easily from it. 


\subsubsection{Data set Seeds}

Seeds data $(N=210, d=7, K=3)$ : this data set consists of 210 patterns belonging to three different varieties of wheat: Kama, Rosa and Canadian. From each species there are 70 observations for area A, perimeter $\mathrm{P}$, compactness $\mathrm{C}\left(C=4^{*}\right.$ pi\& $\left.A / P^{\wedge} 2\right)$, length of kemel, width of kemel, asymmetry coefficient, length of kemel groove[28]. The comparison of algorithms for data set Seeds is listed in Table 8. And the convergence curves of algorithms are shown in Fig.16. In Fig.17, it is the result of anova test for Seeds.

Table 8. Results obtained by the algorithms for 20 different runs on Seeds

\begin{tabular}{ccccc}
\hline Algorithm & Best & Worst & Mean & Std. \\
\hline BPFPA & $\mathbf{3 1 1 . 7 9 7 8}$ & $\mathbf{3 1 1 . 7 9 7 8}$ & $\mathbf{3 1 1 . 7 9 7 8}$ & $\mathbf{2 . 8 E - 1 0}$ \\
FPA & 317.0426 & 335.7757 & 328.0480 & 4.8174 \\
CS & 311.9915 & 313.3699 & 312.6202 & 0.4634 \\
PSO & 311.7979 & 420.1676 & 328.0546 & 39.7004 \\
ABC & 318.0697 & 339.3141 & 327.5305 & 6.2071 \\
DE & 312.4527 & 323.5514 & 316.3921 & 3.9990 \\
K-means & 313.1428 & 313.7343 & 313.4799 & 0.26879 \\
\hline
\end{tabular}

From Table 8, we can discover that the best value, worst value, mean value and standard deviation of BPFPA are the best. And CS performs the second best in all algorithms. It is can be seen clearly that PSO performs the worst. Fig.16 is the convergence curves of algorithms for data set Seeds. The curve of BPFPA is smoother than others can be discovered easily. Faster convergence speed of BPFPA can be found as well. From Fig.17, we can find that the stability of BPFPA, PSO and CS are in really high level.

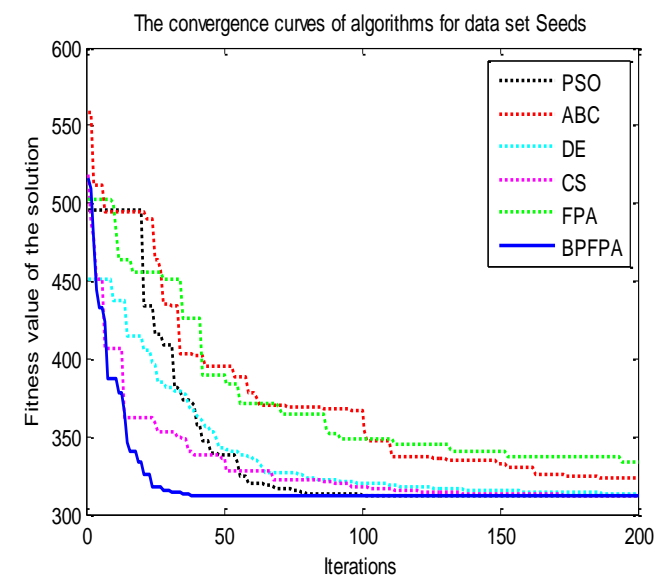

Fig.16 The convergence curves of algorithms for Seeds

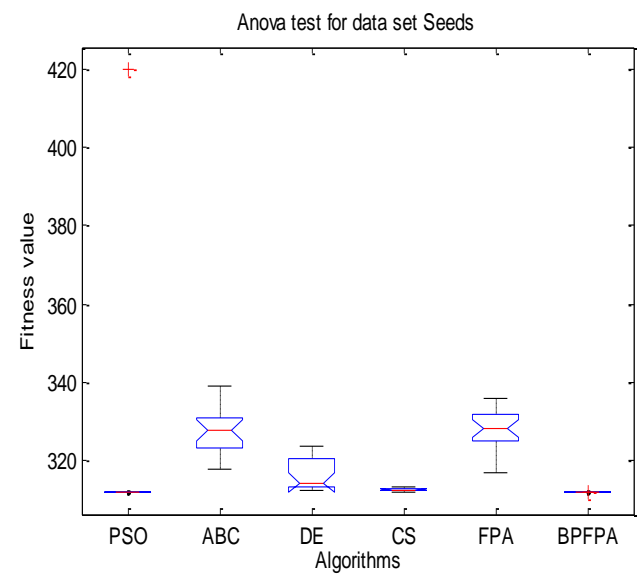

Fig.17 The anova test of algorithms for Seeds

\subsubsection{Data set Haberman's Survival}

Haberman's Survival $(N=306, d=3, K=2)$ : the data set contains cases from a study that was conducted between 1958 and 1970 at the University of Chicago's Billings Hospital on the survival of 
patients who had undergone surgery for breast cancer. It records two survival status patients with age of patient at time of operation, patient's year of operation and number of positive axillary nodes detected [28]. The comparison of algorithms for data set Survival is listed in Table 9. And the convergence curves of algorithms are shown in Fig.18. In Fig.19, it is the result of anova test for Survival.

Table 9. Results obtained by the algorithms for 20 different runs on Survival

\begin{tabular}{ccccc}
\hline Algorithm & Best & Worst & Mean & Std. \\
\hline BPFPA & $\mathbf{2 5 6 6 . 9 8 8 9}$ & 2567.8248 & 2567.0307 & 0.1869 \\
FPA & 2566.9926 & 2567.4245 & 2567.0727 & 0.103246 \\
CS & 2566.9889 & $\underline{2566.9889}$ & $\underline{2566.9889}$ & $\underline{3.492 \mathrm{E}-07}$ \\
PSO & 2566.9889 & 2567.8248 & 2567.1561 & 0.3430 \\
ABC & 2566.9921 & 2567.6142 & 2567.1368 & 0.2107 \\
DE & 2566.9889 & 2569.2452 & 2567.8519 & 0.9110 \\
K-means & 2625.1076 & 3193.5941 & 2655.1274 & 126.7500 \\
\hline
\end{tabular}

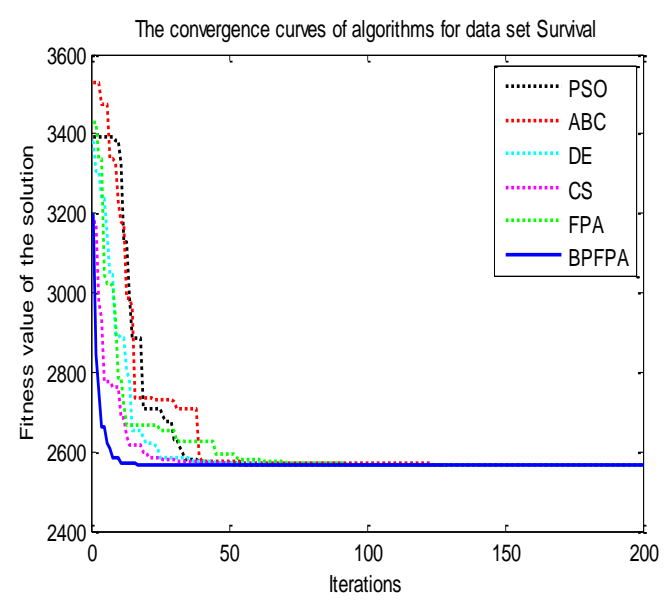

Fig.18 The convergence curves of algorithms for Survival

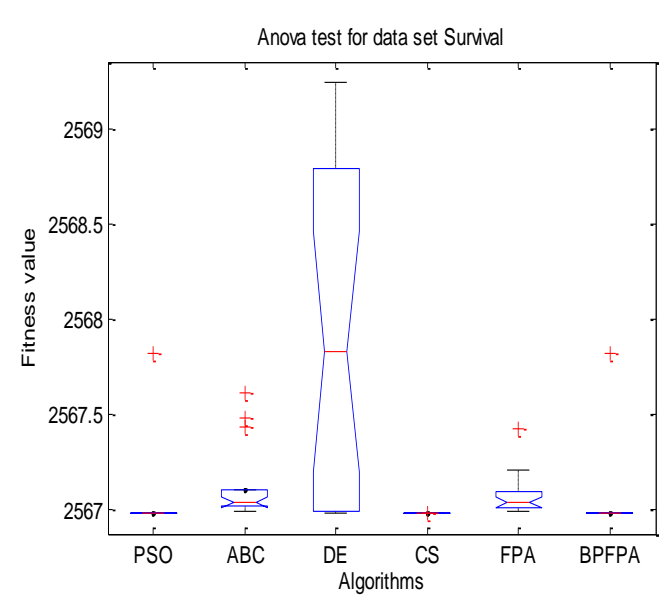

Fig.19 The anova test of algorithms for Survival

From Table 9, best value of BPFPA is the best in mentioned algorithms can be seen. But CS performs the best in worst value, mean value and standard deviation. These three indicators of BPFPA are the second best. Fig.18 shows the convergence curves for data set Survival. It shows the clear fact that BPFPA converges faster than another population based algorithms. High degree stability of BPFPA can be found from Fig.19 easily.

\subsubsection{Data set Wine}

Wine data $(N=178, d=13, K=3)$ : this is the wine data set, which is also taken from MCI laboratory. These data are the results of a chemical analysis of wines grown in the same region in Italy but derived from three different cultivars. The analysis determined the quantities of 13 constituents found in each of the three types of wines. There are 178 instances with 13 numeric attributes in wine data set. All attributes are continuous. There is no missing attribute value [28] [29]. The comparison of algorithms for data set Wine is listed in Table 10. And the convergence curves are shown in Fig.20. In Fig.21, it is the result of anova test for Wine. 
Table 10. Results obtained by the algorithms for 20 different runs on Wine

\begin{tabular}{ccccc}
\hline Algorithm & Best & Worst & Mean & Std. \\
\hline BPFPA & $\mathbf{1 6 2 9 2 . 1 8 4 7}$ & $\mathbf{1 6 2 9 4 . 1 7 2 7}$ & $\mathbf{1 6 2 9 2 . 9 2 3 0}$ & $\mathbf{0 . 7 6 6 3}$ \\
FPA & 16322.0613 & 16381.6074 & 16343.7325 & 16.1486 \\
CS & 16296.4098 & 16306.4835 & 16299.4568 & 2.1980 \\
PSO & 16292.3974 & 16297.6153 & 16294.2251 & 1.1983 \\
ABC & 16391.4522 & 17439.2544 & 16706.4996 & 249.1459 \\
DE & 16336.3502 & 18124.0392 & 16876.5229 & 512.3944 \\
K-means & 16325.1202 & 18436.9520 & 17452.9957 & 1003.6327 \\
\hline
\end{tabular}

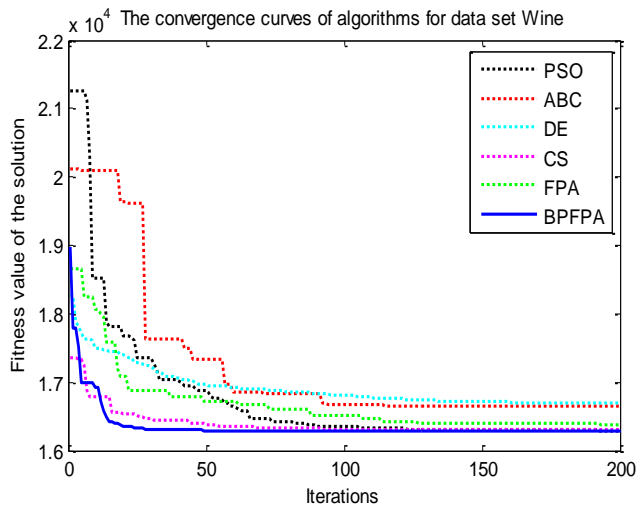

Fig.20 The convergence curves of algorithms for Wine

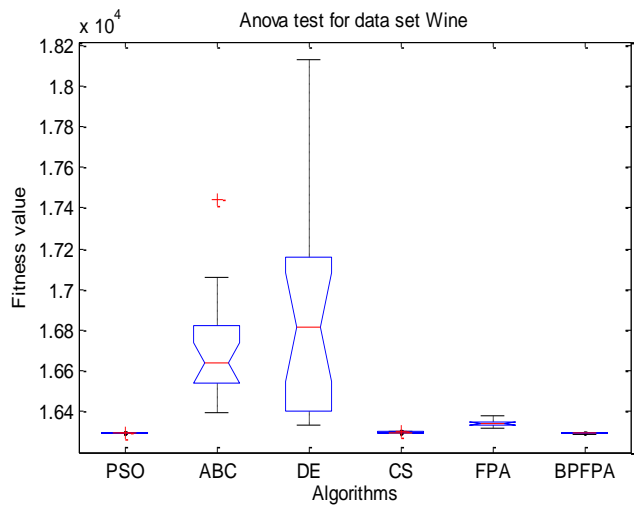

Fig.21 The anova test of algorithms for Wine

We can discover that the best value, worst value, mean value and standard deviation of BPFPA are the best from Table 10. And PSO performs the second best. All of these methods, K-mean may be the worst one for solving clustering problems for data set Wine. Fig.20 shows that the curves of algorithms are all smooth. The curve of BPFPA converges faster than other five. Fig.21 shows that BPFPA, PSO and CS are the algorithms with high level of stability.

\subsection{Graphical Experiment of Clustering}

In section 5.2, large number of numerical simulation experiment is done. Table 1-10 show that BPFPA is an efficient population algorithm for solving clustering problems. Another fact that BPFPA is an algorithm with faster convergence speed and high level of stability can be discovered from Fig.2-21, but the results of numerical experiment are not easy to understand. Therefore, some graphical clustering comparison will be carried out in this section. In subsection 5.3.1, the clustering process of proposed BPFPA is shown. In subsection 5.3.2, five data sets (artificial data set one, artificial data set two, Iris, Contraceptive Method Choice, Survival) are selected to show the graphical result comparison of clustering by proposed BPFPA and another algorithm mentioned here.

\subsubsection{The clustering process of BPFPA}

In this subsection, artificial data set one is selected to show the clustering process of proposed Flower Pollination Algorithm with Bee Pollinator. In order to show the whole process of clustering, the clustering results of BPFPA are selected respectively when iterations are 0,10,15,50, which are shown in Fig.22-25. In these figures, every color represents one class. 


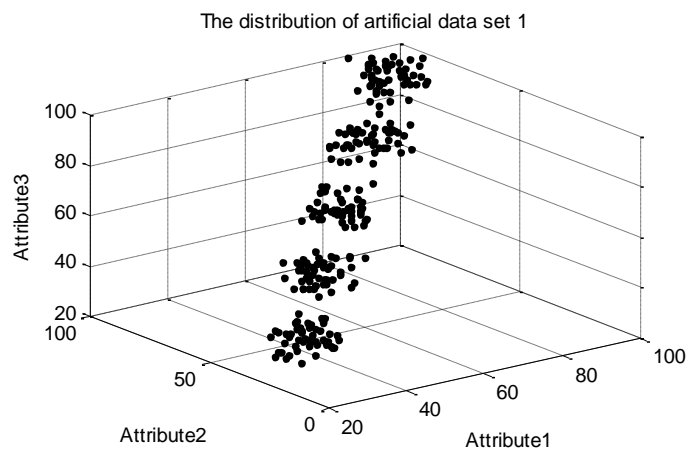

Fig.22 The distribution of artificial data set 1 .

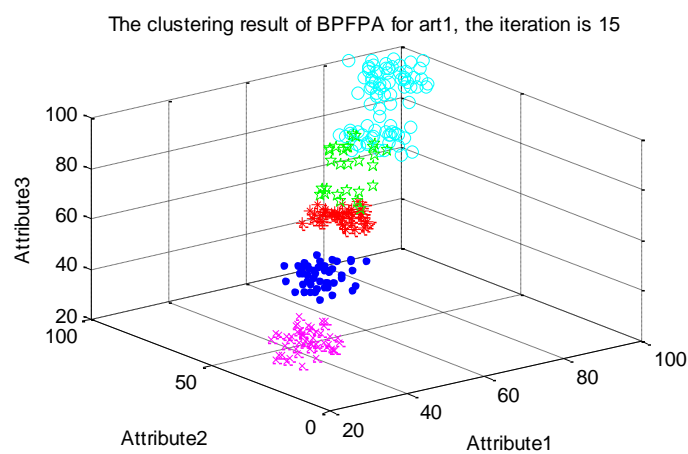

Fig.24 The clustering result of BPFPA for art1, iteration is 15 .

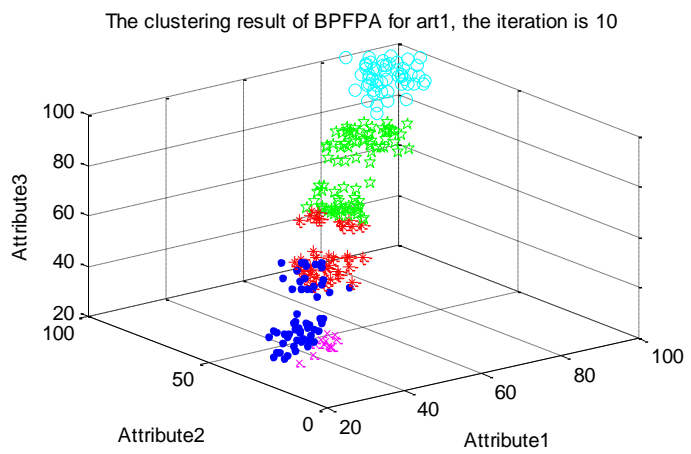

Fig.23 The clustering result of BPFPA for art1, iteration is 10 .

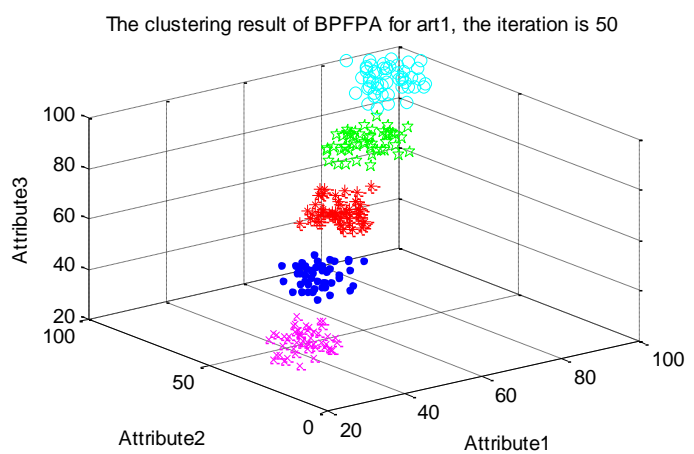

Fig.25 The clustering result of BPFPA for art1, iteration is 50 .

Fig.22 shows the distribution of artificial data set one, Fig.23 is the clustering result when iteration is 10 , chaotic classes are shown in it. Class colored red mingled with the class colored blue, class colored green and class colored red are mixed together. When the iteration is 15, result shown in Fig.24 can be obtained. In Fig.24, the classifications are much clearer than Fig.23. When iteration reaches 50, these five classes are separated successfully by proposed BPFPA, the result is shown in Fig.25.

\subsubsection{Graphical results comparison}

In this subsection, five data sets (artificial data set one, artificial data set two, Iris, Contraceptive Method Choice, Survival) are selected to carried out the graphical comparison between proposed BPFPA and other algorithms. The maximum generations are set to 20 to see the differences between algorithms because of their efficient ability of optimization.

Fig.26 and Fig.27 are the comparison for artificial data set one. BPFPA and CS perform well for art1 from table 1, so CS is selected. In Fig.26, class colored green and the class colored pink mixed together. But in Fig.27, five classes are separated clearly. 

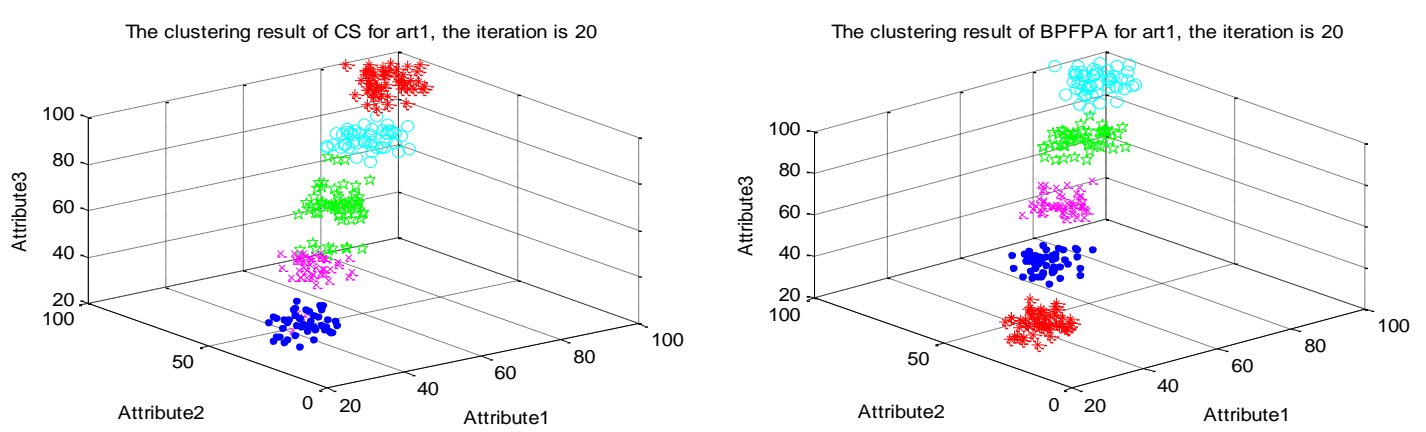

Fig.26 The clustering result of CS for art1, the iteration is 20. Fig.27 The clustering result of BPFPA for art1, the iteration is 20.

Fig.28 and Fig.29 are the comparison for artificial data set two. BPFPA and PSO are selected because of the great performance for artificial data set two, which shows in Table 2. In Fig.28, class colored red mingled with the class colored azure. And the data set is divided into four categories clearly in Fig.29.
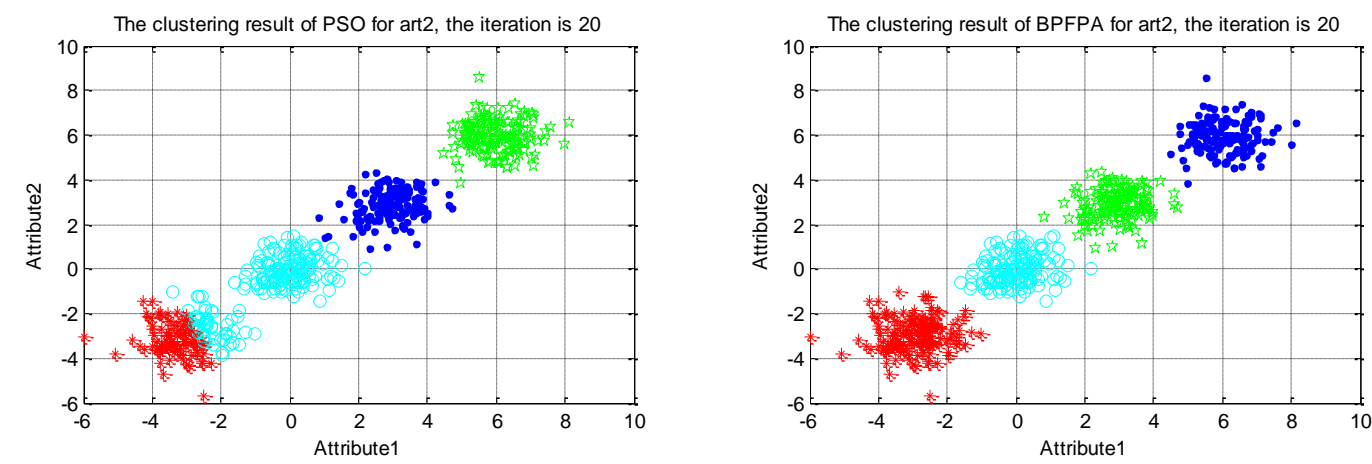

Fig.28 The clustering result of PSO for art2, the iteration is 20. Fig.29 The clustering result of BPFPA for art2, the iteration is 20.

In order to carrying out the comparison for data set Iris, CS and proposed BPFPA are selected, Fig. 30 and Fig.31 are the clustering results of these two population-based algorithms. In Fig.30, two classes those colored red and azure mixed together. The classification of BPFPA (shown in Fig.31) is distinct.
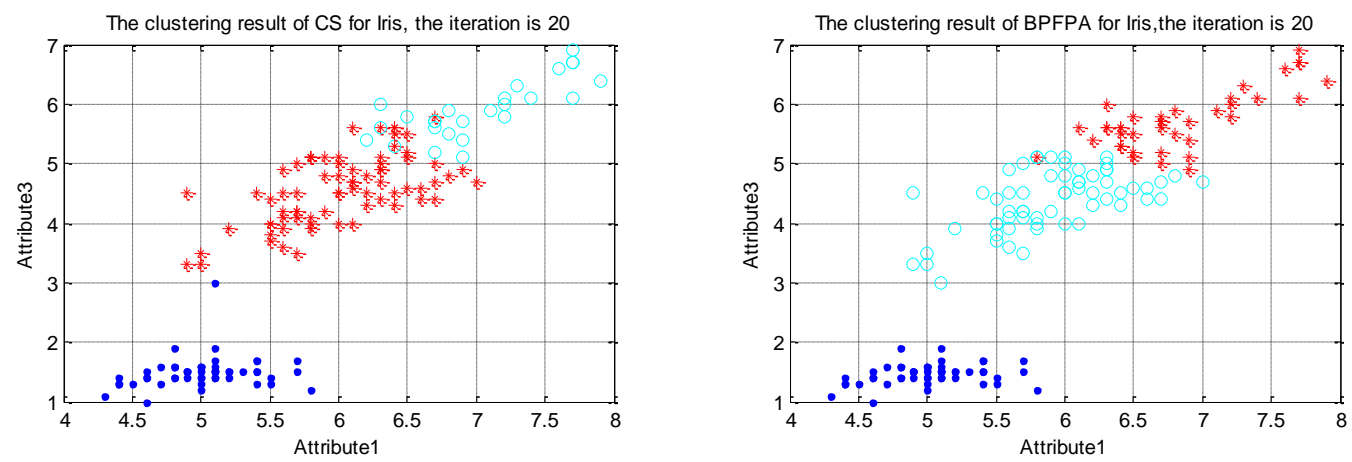

Fig.30 The clustering result of CS for Iris, the iteration is 20. Fig.31 The clustering result of BPFPA for Iris, the iteration is 20

These two figures below show the comparison for data set Contraceptive Method Choice (CMC). From Table 5, we can discover that BPFPA and CS perform better than other algorithms. So CS is selected here to compare with BPFPA. Seen from Fig.32 and Fig.33, it can be found that the clustering ability of BPFPA is slightly better than CS. 

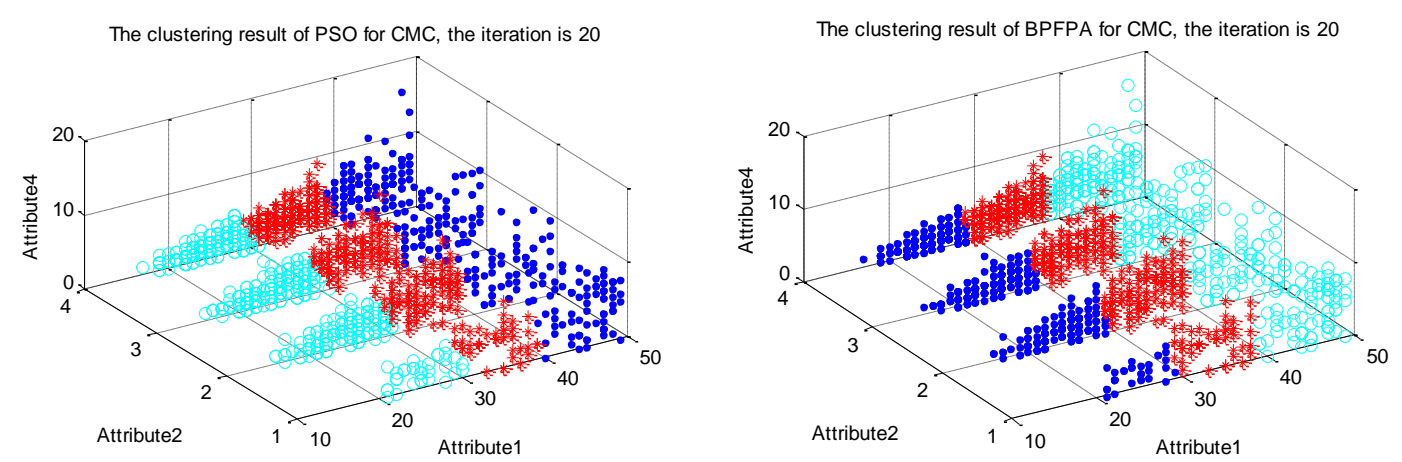

Fig.32 The clustering result of PSO for CMC, iteration is 20. Fig.33 The clustering result of BPFPA for CMC, iteration is 20.

Fig.34 and Fig.35 are the comparison for data set Survival. In Table 9, CS and BPFPA are the better ones for clustering Survival can be easily found. The boundary of two classes is not apparent in Fig.24. And in Fig.35, the two classes are separated apparently.
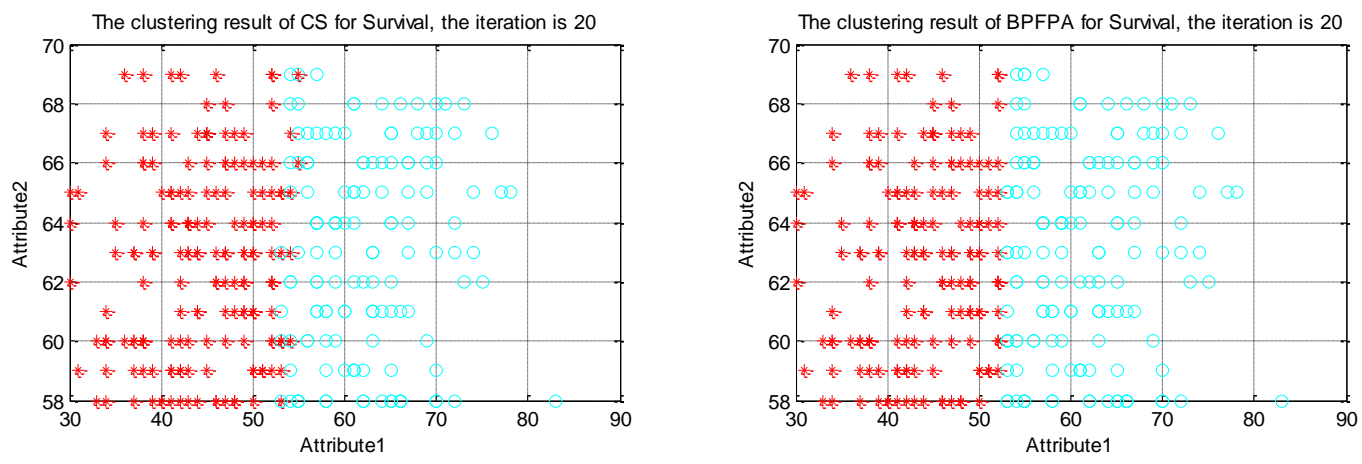

Fig.34 The clustering result of CS for Survival, iteration is 20. Fig.35 The clustering result of BPFPA for Survival, iteration is 20.

\section{Results Analysis}

In section 5, sufficient experiment has been carried out to test the ability of proposed Flower Pollination Algorithm with Bee Pollinator for solving clustering problems. For validating BPFPA comprehensively, ten data sets are used.

Table1-10 shows the numerical result of several mainstream population based algorithms (PSO, DE, $\mathrm{ABC}, \mathrm{CS}, \mathrm{FPA})$. Best value, worst value, mean value and standard deviation are listed respectively in these tables. From these tables, we can discover that BPFPA performs the best in most data set cases. Fig.2-21 shows the comparison of convergence curves and the anova test results for different data sets. From these comparison figures, we can easily find that the convergence speed of BPFPA is faster than other algorithms mentioned in this paper in most cases. Another fact can be found that the stability of BPFPA can reach a relatively high level as well.

In section 5.2, graphical comparisons of algorithms are carried out for five of these data sets (artificial data set one, artificial data set two, Iris, Contraceptive Method Choice, Survival). Fig.22-35 show the graphical comparison results directly. The classification obtained by BPFPA is much clearer than that of other algorithms can be easily found from these figures.

\section{Conclusion and Future Works}

Flower Pollination Algorithm is a new population based algorithm, its outstanding advantage is that it has a strong ability of exploitation. But it often traps in the local minima in the searching process. In 
another words, its exploration ability is not good enough. In this paper, Flower Pollination Algorithm with Bee Pollinator is proposed to overcome the shortage of standard FPA. In global search process, the discard pollen operator is introduced to help jumping out of the local minima, which enhance its exploration ability. In local search process, Elite based mutation operator and crossover operator are used to improve the convergence speed and the diversity of population. In view of the clustering problem, a pollen represents all the centers of clusters. In this paper, two artificial data sets and 8 real instances are selected to compare with other algorithms (k-means, PSO, DE, CS, ABC, FPA). Both numerical experiments results and the graphical experiment results show that Flower Pollination algorithm with Bee Pollinator is more competitive than other algorithms for solving the clustering problem. Future studies will extend the fitness function to also explicitly optimize the intra-cluster distances. More elaborate tests on higher dimensional problems and large number of patterns will be done. The BPFPA clustering algorithm will also be extended to dynamically determine the optimal number of clusters.

\section{Acknowledgements}

This work is supported by National Science Foundation of China under Grants No. 61165015, 6143007. Key Project of Guangxi Science Foundation under Grant No. 2012GXNSFDA053028, Key Project of Guangxi High School Science Foundation under Grant No. 20121ZD008.

\section{References}

[1] R. NG and J.Han. Efficient and effective clustering method for spatial data mining. Proc. 1994 Int. Conf. Very Large Database, Santiago, Chile,1994, pp.144-155.

[2] B. Bhanu, J.Peng. Adaptive integrated image segmentation and object recognition. IEEE Trans. On Systems, Man. And Cybernetics, 2000, 30:427-441.

[3] M R Rao.Cluster Analysis and Mathematical Programming. Journal of the American Statistical Association, 1971, 22: 622-626.

[4] D Fisher. Knowledge Acquisition via Incremental Conceptual Clustering. Machine Learning, 1987,2:139-172,

[5] Chih-Fong Tsai, Yu-Feng Hsu, Chia-Ying Lin, and Wei-Yang Lin. Review: Intrusion detection by machine learning: A review. Expert Syst. Appl, 36:11994-12000, December 2009.

[6] K. R. Zalik. An efficient k-means clustering algorithm. Pattern Recognition Letters. 2008: 1385-1391.

[7] E. H. L. Aarts, J. H. Korst. Simulated annealing and Boltzmann machines. John Wiley and Sons, 1989.

[8] T. Niknam, J. Olamaie, B. Amiri, A hybrid evolutionary algorithm based on ACO and SA for cluster analysis. Journal of Applied Science. 2008, 8 (15):2695-2702.

[9] T. Niknam, B. Bahmani Firouzi, M. Nayeripour. An efficient hybrid evolutionary algorithm for cluster analysis, World Applied Sciences Journal. 2008, 4(2):300-307.

[10] P. S. Shelokar, V. K. Jayaraman, B. D. Kulkarni. An ant colony approach for clustering. Analytica Chimica Acta. 2004, 509(2):187-195.

[11] Kao Y, Cheng K. An ACO-based clustering algorithm. Springer Berlin Heidelberg, 2006:340-347.

[12] Kennedy J, Eberhart R C. Particle swarm optimization. Proc of the IEEE International Conference 
on Neural Networks. Pisca-taway, NJ:IEEE Serivce Center, 1995:1942-1948.

[13] M. Omran, A. P. Engelbrecht, A. Salman. Particle swarm optimization method for image Clustering. International Journal of Pattern Recognition and Artificial Intelligence. 2005,19(3): 297-321.

[14] V. D. Merwe and A. P. Engelbrecht. Data clustering using particle swarm optimization. In Proceedings of IEEE Congress on Evolutionary Computation (CEC03). 2003:215-220.

[15] Kao Y T, Zahara E, Kao I W. A hybridized approach to data clustering. Expert Systems with Applications. 2008, 34(3):1754-1762.

[16] Voges K E, Pope N K L. Rough Clustering Using an Evolutionary Algorithm// System Science (HICSS), $201245^{\text {th }}$ Hawaii International Conference on. IEEE. 2012: 1138-1145.

[17] Yang, X. S(2012), Flower Pollination algorithm for global optimization, in: Unconventional Computation and Natural Computation, Lecture Notes in Computer Science, Vol. 7445, pp. 240-249.

[18] Yang, X. S. Karamanoglu, M., He, X. S. Multiobjective flower algorithm for optimization, Procedia Computer Science, 2013, 18: 861-868.

[19] Marwa Sharawi, E. Emary, Imane Aly Saroit, Hesham El-Mahdy. Flower Pollination Optimization Algorithm for Wireless Sensor Network Lifetime Global Optimization. International Journal of Soft Computing and Engineering, 2014,4:2231-2307.

[20] Osama Abdel Raouf, Ibrahim El-henawy, Mohamed Abdel-Baset. A Novel Hybrid Flower Pollination Algorithm with Chaotic Harmony Search for Solving Sudoku Puzzles. I.J. Modern Education and Computer Science, 2014,3:38-44 Published Online March 2014 in MECS (http://www.mecs-press.org/) DOI: 10.5815/ijmecs.2014.03.05.

[21] Ibrahim El-henawy, Mahmoud Ismail . An Improved Chaotic Flower Pollination Algorithm for Solving Large Integer Programming Problems. International Journal of Digital Content Technology and its Applications,2014, 8(3):

[22] Yang, X. S. Engineering Optimization: An Introduction with Metaheuristic Applications, John Wiley and Sons, USA, 2010.

[23] Karaboga D, Basturk B. A powerful and efficient algorithm for numerical function optimization: artificial bee colony (ABC) algorithm. J. Global Optim, 2007, 39(3):459-471.

[24] Li X, Yin M.An opposition-based differential evolution algorithm for permutation flow shop scheduling based on diversity measure. Adv Eng Softw, 2013, 55:10-31.

[25] R. Storn and K. Price. Differential Evolution - a simple and efficient heuristic for global optimization over continuous spaces. Journal of Global Optimization, 1997,11: 341-359.

[26] J. A. Nelder and R. Mead,. A simplex method for function minimization. Comput. J., 1965.7: 308-313.

[27] X. S. Yang and S. Deb. Cuckoo search via Levy flights. In Proceedings of World Congress on Nature \& Biologically Inspired Computing, pp.210-214, IEEE Publication, New York, NY, USA, 2009.

[28] C. L. Blake. C. J. Merz. UCI Repository of Machine Learning Databases. http://archive.ics.uci.edu/ml/datasets.html

[29] Taher Niknama, Babak Amiri. An efficient hybrid approach based on PSO ACO and k-means for cluster analysis. Appiled Soft Computing. 2010,10(1):183-197. 